\title{
Can the symmetry breaking in the SM be determined by the "second minimum" of the Higgs potential?
}

\author{
Alejandro Cabo, ${ }^{1}$ Jose Carlos Suarez, ${ }^{2}$ Denys Arrebato, ${ }^{3}$ Fernando Guzman, ${ }^{3}$ and Jorge Luis Acosta ${ }^{3}$ \\ ${ }^{1}$ Instituto de Cibernética, Matemática y Física (ICIMAF), \\ La Habana, Cuba. \\ 2 Facultad de Matemáticas y Computación, \\ Universidad de La Habana, La Habana, Cuba. \\ ${ }^{3}$ Instituto de Tecnologías y Ciencias Aplicadas (InSTEC),
} La Habana,Cuba.

\begin{abstract}
The possibility that the spontaneous symmetry breaking in the Standard Model (SM) may be generated by the Top-Higgs Yukawa interaction (which determines the so called "second minimum" in the SM) is investigated. A former analysis about a QCD action only including the Yukawa interaction of a single quark with a scalar field is here extended. We repeat the calculation done in that study of the two loop effective action for the scalar field of the mentioned model. A correction of the former evaluation allowed to select a strong coupling $\alpha\left(\mu, \Lambda_{Q C D}\right)=0.2254 \mathrm{GeV}$ at an intermediate scale $\mu=11.63 \mathrm{GeV}$, in order to fix the minimum of the potential at a scalar mean field determining $175 \mathrm{GeV}$ for the single quark mass. Further, a scalar field mass $m=44 \mathrm{GeV}$ is evaluated, which is also of the order of the experimental Higgs mass. The work is also considering the effects of employing a running with momenta strong coupling. For this purpose, the finite part of the two loop potential contribution determined by the strong coupling, was represented as a momentum integral. Next, substituting in this integral the experimental values of the running coupling, the minimum of the potential curve as a function of the mean field was again fixed to the top quark mass by reducing the scale to the value $\mu=4.95 \mathrm{GeV}$. The consideration of the running coupling also deepened the potential value at the minimum and slightly increased the mass of the scalar field up to $53.58 \mathrm{GeV}$. These results rested in assuming that the low momentum dependence of the coupling is "saturated" to a constant value being close to its experimental value at the lowest momentum measured.
\end{abstract}

Keywords: symmetry breaking, standard model, beyond standard model,

DOI: 10.31526/LHEP.2.2019.98

\section{INTRODUCTION}

The so called "second minimum" of the Higgs field potential in the Standard Model is the result of the Yukawa interaction of the Higgs field with the Top quark. The presence of that minimum had been intrinsically related with the same construction of the model along the years. Special procedures of fixing the various parameters of the theory had to be designed in order to assure that the minimum is separated from the usual Higgs extremum by a potential barrier, being impossible to be tunneled by the standard physical vacuum [1, 2, 3]. In addition, proposals had been advanced that determine the Higgs mass from the condition for the two minima to coincide in values of the potential [3]. In Ref. [4] a simple massless QCD model including only one quark type (modeling the Top quark) and a scalar field (modeling the Higgs field) with a Yukawa interaction between them, was investigated. The aim of the study was to explore a suspicion about that the so called "second minimum" could in fact be responsible for the symmetry breaking in the SM. The idea was to evaluate the two loop effective potential for the scalar field, which in the SM is responsible for the generation of the "second minimum" and to study the possibility of choosing the renormalization conditions to fix the value of the single fermion mass as equal to the top quark one $175 \mathrm{GeV}$. An idea strongly motivating this previous work, came after noting that this additional minimum was identified only after the SM cal- culations arrived up to the two loop order. Then, the question emerges about what could had been the result of an attempt to construct the SM around this new radiative corrections determined minimum, if it would had been known from the start in the SM construction. Up to our knowledge, there had not been attempts to answer this question in the past literature. The results in Ref. [4] were inconclusive, in spite of the fact that the correct experimental values of the Higgs and the Top quark masses were able to be fixed by choosing a definite value of the strong coupling parameter. However, it happened that the calculated value of this parameter was a high one: $\alpha=\frac{g^{2}}{4 \pi}$ close to 1 , which assuming the one loop formula for the relation between the coupling and the scale corresponded to a low momentum scale $\mu=0.49 \mathrm{GeV}$, being outside the region of measured experimental values of the couplings.

In the present work we extend the study done in reference [4]. The discussion starts by considering a new evaluation of the two loop effective potential for the mean value of the scalar field modeling the Higgs. The discussion will proceed in two main directions. 1) The first one is to reconsider the two loop evaluation done in [4] in order to search for possible faults in those calculations, which could had altered the obtained numerical values of the couplings and the scale required for fixing the Top quark mass to its observed value.

2) In second place we will also consider to employ the running values of the coupling with the momentum in the evaluation, in order to check if the decreasing values of the coupling with momentum, also allows to justify the fixing of the potential minimum to reproduce the Top quark mass, which was attained at constant coupling. 
In connection with the new evaluation of the potential, we present the results of the calculation of the three relevant loop integrals determining the effective potential for the scalar field. The revision allowed to detect a numerical error which slightly affected the calculated coupling and scale values for fixing the Top mass. The corrected results were employed to calculate the new values of the coupling and the scale. The change resulted a positive one: the new scale and coupling values (which were assumed to be related by the one loop formula for the coupling) resulted in values being larger for the scale: $\mu=11.63 \mathrm{GeV}$ with respect to the value $\mu=0.49 \mathrm{GeV}$ evaluated in [4]. For coupling values the new result was $\alpha=\frac{g^{2}}{4 \pi}=0.225445$, a smaller result than the high outcome of nearly $\alpha \simeq 1$ following in the former work. Then, this first conclusion supports the suspected possibility that the spontaneous symmetry breaking in the SM could be generated only by the Top quark-Higgs Yukawa interaction.

In order to consider the use of the running coupling with the momentum in evaluating the potential, we first reformulated the finite integral defining the quark-gluon effective potential contribution, which is directly determined by the strong coupling (the quark loop with a contracted gluon propagator). After substracting specially designed divergent parts of the relevant Feynman integral, it was possible to identically transform its finite part in the Minimal Substraction scheme in an integral over the momenta. This technical result directly allowed to substitute the constant strong coupling by running with the momentum one in the integral.

The results assumed that the coupling dependence on the momentum is "saturated" to a constant value for momentum smaller than the smallest of the momenta at which the running coupling is experimentally measured. The calculated effective potential modifies the one evaluated at the initial constant coupling $g\left(\mu, \Lambda_{Q C D}\right)$ at $\mu=11.63 \mathrm{GeV}$. However, after slightly diminishing the scale to the value $\mu=4.95 \mathrm{GeV}$ the minimum of the potential was reinstalled at the top quark mass $175 \mathrm{GeV}$. After this, the value of the potential at the minimum as a function of the mean field is deepened. This result indicates that the diminishing of the coupling with momentum does not drastically alter the behavior of the effective potential, which can be fixed also to retain a minimum at a scalar field mean value imposing a Top quark mass of $175 \mathrm{GeV}$.

Another outcome, is that the new evaluation for the effective potential at constant coupling shows a second derivative at its minimum which predicts a scalar field mass of nearly $m=44 \mathrm{GeV}$. This result is smaller but yet close to the observed Higgs mass of $126 \mathrm{GeV}$. The new value corrects the one calculated in Ref. [4]. The consideration of the running coupling only increases the scalar field mass up to a close value of $53.58 \mathrm{GeV}$.

We estimate this conclusion as one interesting outcome of the analysis. It means that once the Top quark mass is fixed, the spontaneous symmetry breaking pattern associated to the TopHiggs Yukawa interaction (that is to the "second minimum") is able to determine a mass value of the scalar field being close to the experimentally measured mass of the Higgs particle. Therefore, it might be expected that the many new contributions to the curvature of the Higgs potential that will exist in a more realistic SM type of calculation make feasible to fix the experimental value of $126 \mathrm{GeV}$ for the Higgs mass. A first possibility for it is offered by the required presence of a squared in the Higgs field Lagrangian term. This term may be independently needed by renormalization and the smallness of the evaluated contribution to the Higgs mass may allow to fix a positive sign for the mass squared factor multiplying the squared Higgs field.

Therefore, the discussion in the work still sustain the expectation about the possibility of describing the full SM after considering an initial Lagrangian in which the classical Mexican hat potential may be absent. The exploration of this possibility will be considered elsewhere.

The plan of the work is as follows. In Section 2, the model and its Feynman expansion are reviewed. Section 3 continues by presenting the new evaluation of the effective potential for the mean scalar, and discussing the changes with respect to the previous calculations in Ref. [4]. Section 4 exposes the determination of the new values of the scale parameter $\mu=11.63 \mathrm{GeV}$ and its associated strong coupling value which allowed to fix the Top mass as equal to the experimental value. Next, Section 5 describes the derivation of the transformation of the effective potential contribution depending on the strong coupling, in a momentum integral. This allows to substitute the constant value of the strong coupling by the running with the momentum formula in Section 6. Finally, in Section 7 some physical connections of the simple model considered here with the SM are discussed. The results are reviewed at the Summary.

\section{THE MODEL}

Let us now start by reviewing the main elements of the model discussed in [4]. The generating functional of the Feynamn expansion is based in an action including a singlet scalar field interacting with only one type of quark. The functional was chosen in the form

$$
\begin{aligned}
Z[j, \eta, \bar{\eta}, \xi, \bar{\zeta}, \rho] & =\frac{1}{\mathcal{N}} \int \mathcal{D}[A, \bar{\Psi}, \Psi, \bar{c}, c, \phi] \times \\
& \times \exp [i S[A, \bar{\Psi}, \Psi, \bar{c}, c, \phi]] .
\end{aligned}
$$

The action was taken in the form written below, in which in addition to the usual massless QCD Lagrangian, there were only considered a Yukawa interaction term of a quark with a one component scalar field and the corresponding action term for the scalar. To simplify the discussion, the free action of the scalar field was defined as a massless free term in the absence of self-interaction. The action, after decomposed in its free and interaction parts, is written below

$$
\begin{aligned}
S= & \int d x\left(\mathcal{L}_{0}+\mathcal{L}_{1}\right), \\
\mathcal{L}_{0}= & \mathcal{L}^{g}+\mathcal{L}^{g h}+\mathcal{L}^{q}+\mathcal{L}^{\phi}, \\
\mathcal{L}^{g}= & -\frac{1}{4}\left(\partial_{\mu} A_{v}^{a}-\partial_{\nu} A_{\mu}^{a}\right)\left(\partial^{\mu} A^{a, v}-\partial^{v} A^{a, \mu}\right)- \\
& \frac{1}{2 \alpha}\left(\partial_{\mu} A^{\mu, a}\right)\left(\partial^{v} A_{v}^{a}\right), \\
\mathcal{L}^{g h}= & \left(\partial^{\mu} \chi^{* a}\right) \partial_{\mu} \chi^{a}, \\
\mathcal{L}^{q}= & \bar{\Psi} i \gamma^{\mu} \partial_{\mu} \Psi, \\
\mathcal{L}^{\phi}= & \frac{1}{2} \partial^{\mu} \phi \partial_{\mu} \phi,
\end{aligned}
$$




$$
\begin{aligned}
\mathcal{L}_{1}=- & \frac{g}{2} f^{a b c}\left(\partial_{\mu} A_{v}^{a}-\partial_{\nu} A_{\mu}^{a}\right) A^{b, \mu} A^{c, v}- \\
& g^{2} f^{a b e} f^{c d e} A_{\mu}^{a} A_{v}^{b} A^{c, \mu} A^{d, v}- \\
& g f^{a b c}\left(\partial^{\mu} \chi^{* a}\right) \chi^{b} A_{\mu}^{c}+ \\
& g \bar{\Psi} T^{a} \gamma^{\mu} \Psi A_{\mu}^{a}+y \bar{\Psi} \phi .
\end{aligned}
$$

The dimensionless Yukawa coupling $y$ will be assumed to have a value close to $y=1$ as it had been estimated in the literature [6]. After constructing the Feynman expansion being associated to the above generating function and classical action, the evaluation of the effective potential as a function of an homogeneous scalar (Higgs resembling) field was considered in reference [4], up to the two loop approximation. All the notations for the quantum fields quantities, Minkowski metric, etc. used in this work closely follow the ones employed in reference [5] .

\section{TWO LOOPS EFFECTIVE POTENTIAL OF THE SCALAR FIELD}

Let us consider again the evaluation of all the contributions to the effective potential $V(\phi)$ for the scalar field $\phi$, up to the two loop order. This is the quantity determining the spontaneous symmetry breaking effect in the considered model and checking its calculation is central for to be sure about its physical predictions. We will see that numerical errors slightly affected the results of the previous work. The corrections will then allow to modify the results for the scale parameter $\mu$ and the coupling $g\left(\mu, \Lambda_{Q C D}\right)$ values required for fixing the Top quark mass value for the fermion in the model.

\subsection{The one loop term}

The analytic expression for the one loop contribution shown in Fig. 1 which was evaluated in [4], was given by the classical logarithm of the fermion quark determinant as:

$$
\begin{aligned}
\Gamma^{(1)}[\phi] & =-V^{(D)} N \int \frac{d p^{D}}{i(2 \pi)^{D}} \times \\
& \log \left[\operatorname{Det}\left(G_{i i^{\prime}}^{(0) r r^{\prime}}(\phi, p)\right)\right], \\
D & =4-2 \epsilon,
\end{aligned}
$$

where $D$ is the space dimension of dimensional regularization and the free fermion propagator was written as before in the conventions of Ref. [5], which, as mentioned before, will be used also throughout this work. This propagator is defined as

$$
\begin{aligned}
G_{i i^{\prime}}^{(0) r r^{\prime}}(\phi, p) & =\delta^{i i^{\prime}}\left(\frac{1}{-p_{\mu} \gamma^{\mu}+\phi}\right)^{r r^{\prime}} \\
& =-\frac{\delta^{i i^{\prime}}}{p^{2}-\phi^{2}}\left(p_{\mu} \gamma^{\mu}+\phi\right)^{r r^{\prime}} .
\end{aligned}
$$

As before, assuming the case of QCD with $S U(N)$ symmetry for $N=3$, and evaluating the spinor and color traces, the one loop expression is simplified to become

$$
\Gamma^{(1)}[\phi]=V^{(D)} \frac{N}{2} \int \frac{d p^{D}}{i(2 \pi)^{D}} \log \left[\left(\phi^{2}-p^{2}\right)^{4}\right] .
$$

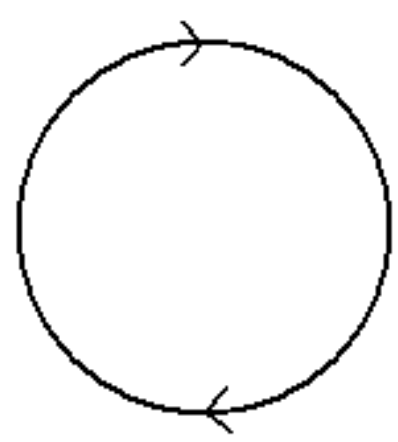

FIGURE 1: It shows the quark one loop correction. The result depends on the scalar "mass" field $\phi$ through the quark free propagator which is the usual free Green function of QCD, in which the mass is substituted by $\phi$.

Taking the derivative over $\phi^{2}$ of $\Gamma^{(1)}[\phi]$ allows to write the easily integrable expression

$$
\frac{d}{d \phi^{2}} \Gamma^{(1)}[\phi]=V^{(D)} 2 N \int \frac{d p^{D}}{(2 \pi)^{D}} \frac{1}{\left(p^{2}+\phi^{2}\right)} .
$$

Making use of the identity

$$
\int \frac{d p^{D}}{(2 \pi)^{D}} \frac{1}{\left(p^{2}+\lambda^{2}\right)}=\frac{\Gamma\left(1-\frac{D}{2}\right)}{(4 \pi)^{\frac{D}{2}}}\left(\lambda^{2}\right)^{\frac{D}{2}-1},
$$

and integrating the result back over $\phi^{2}$, gives the dimensionally regularized expression

$$
\begin{aligned}
\Gamma^{(1)}[\phi] & =V^{(D)} \frac{2 N \Gamma\left(1-\frac{D}{2}\right)}{\left(\frac{D}{2}\right)(4 \pi)^{\frac{D}{2}}}\left(\phi^{2}\right)^{\frac{D}{2}-1} \\
& =V^{(D)} \frac{2 N \Gamma(\epsilon-1)}{\left(\frac{D}{2}\right)(4 \pi)^{2-\epsilon}}\left(\phi^{2}\right)^{2-\epsilon},
\end{aligned}
$$

which coincides with the corresponding expression in [4]. Let us divide $\Gamma^{(1)}[\phi]$ by $\frac{V(D)}{\mu^{2 \epsilon}}$, in order to write the action density. The quantity $\mu$ in the denominator is the dimensional regularization scale parameter, and the divisor $\mu^{2 \epsilon}$, which tends to one on removing the regularization, is introduced in order to avoid results containing logarithms of quantities having dimension. Then, the one loop Lagrangian density takes the form

$\gamma^{(1)}[\phi]=\frac{\Gamma^{(1)}[\phi]}{\frac{V(D)}{\mu^{2 \epsilon}}}=\frac{2 N \Gamma(\epsilon-1)}{\left(\frac{D}{2}\right)(4 \pi)^{2-\epsilon}} \phi^{4}\left(\frac{\phi}{\mu}\right)^{-2 \epsilon}$,

also coinciding with former result in [4]. After deleting the pole part of the above expression according to the Minimal Substraction rule, and taking the limit $\epsilon \rightarrow 0$, gives the finite part of the one loop action density as

$$
\begin{gathered}
{\left[\gamma^{(1)}[\phi]\right]_{\text {finite }}^{\epsilon \rightarrow 0}=\frac{3 \phi^{4}}{32 \pi^{2}}(-3+2 \gamma-4 \log (2)-} \\
\left.2 \log (\pi)+2 \log \left(\frac{\phi^{2}}{\mu^{2}}\right)\right)
\end{gathered}
$$

where $\gamma=0.57721 .$. is the Euler constant. 
Finally, the one loop potential energy density is given by the negative of the above quantity

$$
\begin{aligned}
v^{(1)}[\phi]= & -\frac{3 \phi^{4}}{32 \pi^{2}}(-3+2 \gamma-4 \log (2)- \\
& \left.2 \log (\pi)+2 \log \left(\frac{\phi^{2}}{\mu^{2}}\right)\right), \\
= & -\frac{3 \phi^{4}}{32 \pi^{2}}\left(-3+2 \gamma+2 \log \left(\frac{\phi^{2}}{4 \pi \mu^{2}}\right)\right) .
\end{aligned}
$$

It can be noticed that one loop potential density is unbounded from below for increasing values of the scalar field, which is its main property determining the dynamical generation of the field $\phi$ in the model.

\subsection{Quark-gluon two loop term}

Let us start now evaluating the two loop quark-gluon term which was calculated in reference [4] and is illustrated in Fig. 2. Again, after evaluating the color and spinor traces the analytic expression for this contribution was obtained in a coinciding form as follows

$$
\begin{gathered}
\Gamma_{g}^{(2)}[\phi]=-V^{(D)} g^{2}\left(N^{2}-1\right) \int \frac{d p^{D} d q^{D}}{i^{2}(2 \pi)^{2 D}} \times \\
\frac{D \phi^{2}-(D-2) p \cdot(p+q)}{q^{2}\left(p^{2}-\phi^{2}\right)\left((p+q)^{2}-\phi^{2}\right)}
\end{gathered}
$$

where, as before $g^{2}$ is the QCD coupling constant in the dimensional regularization scheme, which introduces the scale parameter $\mu$ according to

$g=g_{0} \mu^{2-\frac{D}{2}}=g_{0} \mu^{\epsilon}$

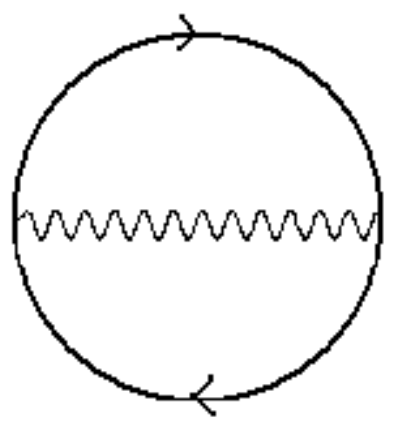

FIGURE 2: The two loop contribution determined by the strong interaction. As before, the $\phi$ dependence of the result is introduced though the free quark propagator.

After repeating the same steps followed in [4], that is, symmetrizing the expression of $\Gamma_{g}^{(2)}[\phi]$ under the change of sign in the momentum $q$, by means of the integration variable shift $p \rightarrow p-\frac{q}{2}$ and the use of the identity

$$
\begin{gathered}
p^{2}=\left(p+\frac{q}{2}\right)^{2}-\phi^{2}+\phi^{2}- \\
\frac{q^{2}}{4}-q \cdot p
\end{gathered}
$$

the quark-gluon term is written in the form

$\Gamma_{g}^{(2)}[\phi]=\Gamma_{g}^{(2.1)}[\phi]+\Gamma_{g}^{(2,2)}[\phi]$,

where $\Gamma_{g}^{(2,1)}[\phi]$ and $\Gamma_{g}^{(2,2)}[\phi]$ have the formulae

$$
\begin{aligned}
\Gamma_{g}^{(2,1)}[\phi]= & -V^{(D)} 2 \phi^{2} g^{2}\left(N^{2}-1\right) \int \frac{d k_{1}^{D} d k_{2}^{D}}{i^{2}(2 \pi)^{2 D}} \times \\
& \frac{1}{k_{1}^{2}\left(k_{2}^{2}-\phi^{2}\right)\left(\left(k_{1}+k_{2}\right)^{2}-\phi^{2}\right)} \\
= & -V^{(D)} \frac{2 \phi^{2} g^{2}\left(N^{2}-1\right)}{i^{2}(2 \pi)^{2 D}} J_{111}(0, \phi, \phi), \\
\Gamma_{g}^{(2,2)}[\phi]= & -V^{(D)} \frac{(D-2) g^{2}\left(N^{2}-1\right)}{2 i^{2}(2 \pi)^{2 D}} \times \\
& \left(\int d k_{1}^{D} \frac{1}{k_{1}^{2}-\phi^{2}}\right)^{2},
\end{aligned}
$$

in which, the master two loop integral $J_{111}(0, \phi, \phi)$ was evaluated making use of the results in Ref. [7], and its explicit form for the particular values of our arguments is:

$$
\begin{aligned}
J_{111}(0, \phi, \phi) & =\int d k_{1}^{D} d k_{2}^{D} \times \\
& \frac{1}{k_{1}^{2}\left(k_{2}^{2}-\phi^{2}\right)\left(\left(k_{1}+k_{2}\right)^{2}-\phi^{2}\right)} \\
& =-\frac{A(\epsilon) \pi^{4-2 \epsilon}}{\epsilon^{2}}\left(\phi^{2}\right)^{1-2 \epsilon}, \\
A(\epsilon) & =\frac{(\Gamma(1+\epsilon))^{2}}{(1-\epsilon)(1-2 \epsilon)} .
\end{aligned}
$$

Then, using the above definitions the following expression can be written for $\Gamma_{g}^{(2,1)}$

$$
\begin{gathered}
\Gamma_{g}^{(2,1)}[\phi]=-\frac{V^{(D)} 2 g_{0}^{2} \mu^{2 \epsilon}\left(N^{2}-1\right)}{(2 \pi)^{8-4 \epsilon}} \times \\
\frac{A(\epsilon) \pi^{4-2 \epsilon}}{\epsilon^{2}} \phi^{4}\left(\phi^{2}\right)^{-2 \epsilon},
\end{gathered}
$$

and coincides with the former result.

However, for the case of $\Gamma_{g}^{(2,2)}$, we found that the result in [4] included an error after the square of the one loop integral in equation (11) was substituted: a minus sign coming from the Wick rotation of the squared momentum integral in (21) was omitted. Thus, the correct expression for this term should be

$$
\begin{gathered}
\Gamma_{g}^{(2,2)}[\phi]= \\
\pi^{4-2 \epsilon}\left(\Gamma_{g}(\epsilon) \frac{g_{0}^{2} \mu^{2 \epsilon}\left(N^{2}-1\right) 2(1-\epsilon)}{2(2 \pi)^{8} \phi^{4}\left(\phi^{2}\right)^{-2 \epsilon}} \times\right.
\end{gathered}
$$

Again dividing by $\frac{V(D)}{\mu^{2 \epsilon}}$ to evaluate the action densities gives

$$
\begin{aligned}
\gamma_{g}^{(2,1)}[\phi]= & \frac{\Gamma_{g}^{(2,1)}[\phi]}{\frac{V(D)}{\mu^{2 \epsilon}}} \\
= & -\frac{2 g_{0}^{2}\left(N^{2}-1\right)}{(2 \pi)^{2 D}} \times \\
& \frac{A(\epsilon) \pi^{4-2 \epsilon}}{\epsilon^{2}} \phi^{4}\left(\frac{\phi^{2}}{\mu^{2}}\right)^{-2 \epsilon},
\end{aligned}
$$


and for the corrected term

$$
\begin{aligned}
\gamma_{g}^{(2,2)}[\phi]= & \frac{\Gamma_{g}^{(2,2)}[\phi]}{\frac{V(D)}{\mu^{2 \epsilon}}} \\
= & -V^{(D)} \frac{g_{0}^{2} \mu^{2 \epsilon}\left(N^{2}-1\right) 2(1-\epsilon)}{2(2 \pi)^{8-4 \epsilon}} \times \\
& \pi^{4-2 \epsilon}(\Gamma(\epsilon-1))^{2} \phi^{4}\left(\phi^{2}\right)^{-2 \epsilon} .
\end{aligned}
$$

Therefore, repeating the process of substracting the divergent poles and taking the limit $\epsilon \rightarrow 0$, the total quark-gluon two loop finite contribution to the action density takes the expression

$$
\begin{aligned}
{\left[\gamma_{g}^{(2)}[\phi]\right]_{\text {finite }}^{\epsilon \rightarrow 0} } & =-\frac{g_{0}^{2}}{64 \pi^{4}} \phi^{4}\left(30-28 \gamma+12 \gamma^{2}+\right. \\
& \pi^{2}+56 \log (2)-48 \gamma \log (2)+ \\
& 48 \log (2)^{2}+28 \log (\pi)-24 \gamma \log (\pi)+ \\
& 48 \log (2) \log (\pi)+12 \log (\pi)^{2}+ \\
& (24 \gamma-28-48 \log (2)-48 \log (\pi)) \times \\
& \left.\log \left(\frac{\phi^{2}}{\mu^{2}}\right)+12\left(\log \left(\frac{\phi^{2}}{\mu^{2}}\right)\right)^{2}\right) \\
& =-v_{g}^{(2)}[\phi],
\end{aligned}
$$

in which $v_{g}^{(2)}[\phi]$ defines the quark-gluon contribution to the effective potential.

It should be remarked, that the leading logarithm squared term in the action is negative, indicating that the contribution of the usual quark-gluon diagram to the potential (equal to minus the action) up to the two loop approximation remains being bounded from below as a function of $\phi$, after the corrections are done.

The divergent contribution to the action follows in the form

$$
\begin{aligned}
\gamma_{g, \operatorname{div}}^{(2)}[\phi] & =-\frac{3 g^{2} \phi^{4}}{32 \pi^{4} \epsilon^{2}}+\frac{g^{2} \phi^{4}}{32 \pi^{4} \epsilon} \times \\
& \left(-7+6 \gamma-6 \log (4 \pi)+12 \log \left(\frac{\phi}{\mu}\right)\right)
\end{aligned}
$$

which defines the Minimal Substraction making finite the quark-gluon two loops contribution.

\subsection{Scalar-quark two loop term}

Finally, let us repeat the evaluation of the two loop term being associated to the quark-scalar loop illustrated in Fig. 3. Due to the absence of spinor and color structures in the vertices, the analytic expression for this term is again calculated to be

$$
\begin{aligned}
\Gamma_{Y}^{(2)}[\phi]= & V^{(D)} 2 N \int \frac{d p^{D} d q^{D}}{i^{2}(2 \pi)^{2 D}} \times \\
& \frac{p^{2}-\frac{q^{2}}{4}+\phi^{2}}{q^{2}\left(\left(p+\frac{q}{2}\right)^{2}-\phi^{2}\right)\left(\left(p-\frac{q}{2}\right)^{2}-\phi^{2}\right)}
\end{aligned}
$$

which in a close way as it was done for the quark-gluon term, was evaluated in the form

$$
\begin{aligned}
\Gamma_{Y}^{(2)}[\phi] & =V^{(D)} \frac{4 N}{i^{2}(2 \pi)^{2 D}} J_{111}(0, \phi, \phi) \\
& -V^{(D)} N\left(\int \frac{d k_{1}^{D}}{i(2 \pi)^{D}} \frac{1}{k_{1}^{2}-\phi^{2}}\right)^{2} \\
& =\frac{V^{(D)} 4 N}{(2 \pi)^{8-4 \epsilon}} \frac{A(\epsilon) \pi^{4-2 \epsilon}}{\epsilon^{2}} \phi^{4}\left(\phi^{2}\right)^{-2 \epsilon} \\
& -V^{(D)} \frac{N}{(2 \pi)^{8-4 \epsilon}} \pi^{4-2 \epsilon} \times \\
& (\Gamma(\epsilon-1))^{2} \phi^{4}\left(\phi^{2}\right)^{-2 \epsilon} .
\end{aligned}
$$

It can be noted that the imaginary number included in the squared momentum integral, was now and before properly considered, avoiding in this way the error done in the former evaluation of the quark-gluon term. The division by the volume

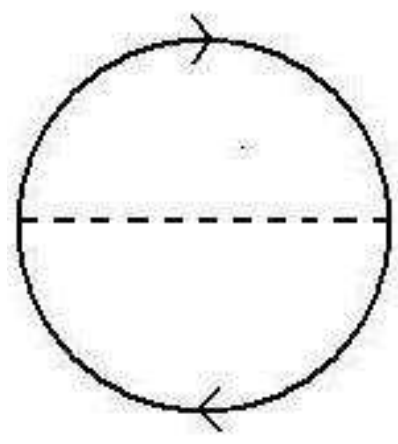

FIGURE 3: The two loop contribution determined by the scalarquark interaction. The Yukawa coupling between the quark and the scalar field was chosen as approximately equal to one.

$\frac{V(D)}{\mu^{2 \epsilon}}$ again allows to write for the action density, the formula

$$
\begin{aligned}
\gamma_{Y}^{(2)}[\phi] & =\frac{4 N}{(2 \pi)^{8-4 \epsilon}} \frac{A(\epsilon) \pi^{4-2 \epsilon}}{\epsilon^{2}} \phi^{4}\left(\frac{\phi^{2}}{\mu^{2}}\right)^{-2 \epsilon} \\
& -\frac{N}{(2 \pi)^{8-4 \epsilon}} \pi^{4-2 \epsilon}(\Gamma(\epsilon-1))^{2} \phi^{4}\left(\frac{\phi^{2}}{\mu^{2}}\right)^{-2 \epsilon}
\end{aligned}
$$

Substracting the divergent pole part in $\epsilon$, passing to the limit $\epsilon \rightarrow 0$ gives for the potential density

$$
\begin{aligned}
v_{Y}^{(2)}[\phi] & =-\left[\gamma_{Y}^{(2)}[\phi]\right]_{\text {finite }}^{\epsilon \rightarrow 0} \\
& =-\frac{3}{512 \pi^{4}} \phi^{4}\left(50-40 \gamma+12 \gamma^{2}+\pi^{2}+\right. \\
& 96 \log (2)-64 \gamma \log (2)+ \\
& 64 \log (2)^{2}+48 \log (\pi)-32 \gamma \log (\pi)+ \\
& 64 \log (2) \log (\pi)+16 \log (\pi)^{2}- \\
& 8 \log (4 \pi)+8 \gamma \log (4 \pi)-4 \log (4 \pi)^{2}+ \\
& (24 \gamma-40-64 \log (2)-32 \log (\pi)+ \\
& \left.8 \log (4 \pi)) \log \left(\frac{\phi^{2}}{\mu^{2}}\right)+12\left(\log \left(\frac{\phi^{2}}{\mu^{2}}\right)\right)^{2}\right) .
\end{aligned}
$$


It can be noted that this contribution, being a two loop one, also includes a squared logarithm term. However, its sign is contrary to the one appearing in the quark gluon loop.

For the total two loop effective potential it follows

$V[\phi, \mu]=v^{(1)}[\phi]+v_{g}^{(2)}[\phi]+v_{Y}^{(2)}[\phi]$.

\section{FIXING THE POTENTIAL MINIMUM FOR $M_{\text {TOP }}=175 \mathrm{GEV}$}

Let us consider the sum of all the just evaluated contributions to the potential energy density $V(\phi)$. Its expression is a combination of terms of the form $\phi^{4}, \phi^{4} \log \left(\frac{\phi}{\mu}\right)$ and $\phi^{4}\left(\log \left(\frac{\phi}{\mu}\right)\right)^{2}$, with coefficients that only depend on the strong coupling $g_{0}$ in the present first analysis. Then, in order to approach the physical situation, we evaluated the potential $V(\phi)$ at the values of $g_{0}$ satisfying the one loop formula for the running coupling constant [5]

$g_{0}\left(\mu, \Lambda_{Q C D}\right)=2 \sqrt{\frac{2}{7}} \pi \sqrt{\frac{1}{\log \left(\frac{\mu}{\Lambda_{Q C D}}\right)}}$.

The $\Lambda_{Q C D}$ constant was chosen to be the estimated $\Lambda_{Q C D}=$ $0.217 \mathrm{GeV}$. Here it should be remarked that for the determination of the one loop coupling we have assumed the number of fermions as equal to six, in place of one, as it is proper for the model under discussion. This criterion was adopted in order to assume the strong coupling as more representative of the situation in the SM. In spite of this, we also had evaluated the results for the case $N_{f}=1$ and the qualitative conclusions of the work did not appreciably changed.

Next, we studied the potential curves in order to examine the behavior of their minimum as functions of $\phi$, when the scale $\mu$ is changed. It follows that when the strong coupling starts to increase as the scale diminish down to one $\mathrm{GeV}$, the value of $\phi$ at the minima, which determines the quark mass also decreases. For the particular value of $\mu=11.63 \mathrm{GeV}$, the potential curve is shown in Fig. 4 . The particular value of $\mu$ chosen, fixes the position of the minimum at a field $\phi$ defining a quark mass of $175 \mathrm{GeV}$. The set of parameters for this curve are

$$
\begin{aligned}
\mu & =11.63 \mathrm{GeV}, \\
g_{0} & =1.68316 \quad\left(\alpha=\frac{\left(g_{0}\right)^{2}}{4 \pi}=0.225445\right), \\
\Lambda_{Q C D} & =0.217 \mathrm{GeV} .
\end{aligned}
$$

\subsection{The mass of the scalar field}

Let us consider the mass of the scalar field to be defined in the present calculation. For evaluating it, we write the following approximate two loop action for the scalar field linear propagation modes around the minimum of the potential at the mean field homogeneous value $\phi$

$$
\begin{aligned}
\mathcal{L}^{\phi} & =\frac{1}{2} \partial^{\mu} \delta \phi \partial_{\mu} \delta \phi-\frac{1}{2} \delta \phi V^{\prime \prime}[\phi] \delta \phi, \\
V^{\prime \prime}[\phi] & =\left.\frac{\partial^{2}}{\partial \phi^{2}} V[\phi+\delta \phi]\right|_{\delta \phi=0} .
\end{aligned}
$$

The Lagrange equation for the propagating scalar field waves $\delta \phi=\exp (-i p \cdot x)$ then writes

$\left(\partial^{\mu} \partial_{\mu} \phi+V^{\prime \prime}[\phi]\right) \delta \phi=\left(-p^{2}+V^{\prime \prime}[\phi]\right) \delta \phi=0$.

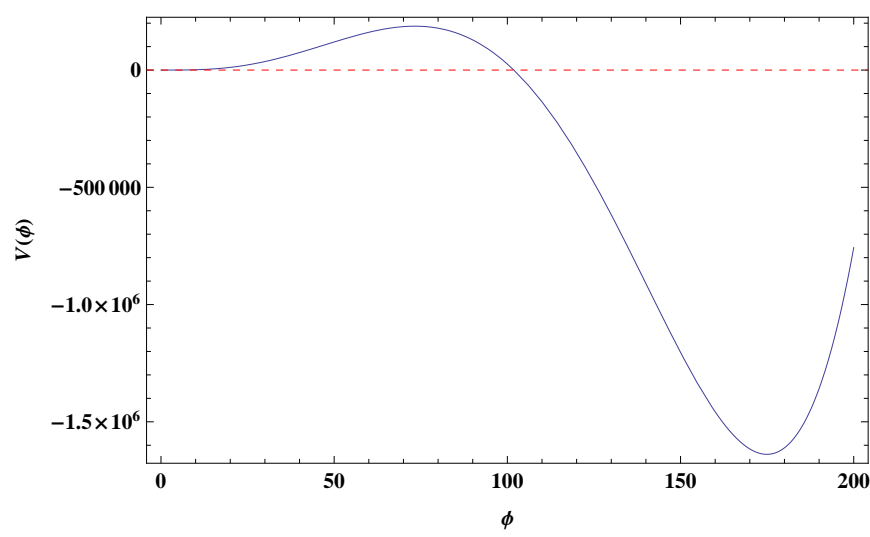

FIGURE 4: The effective potential of the mean field $\phi$ for the value of the scale $\mu$ determining $\phi$ at the potential minimum being equal to the top quark mass $m_{\text {top }}=175 \mathrm{GeV}$. The second derivative at the minimum gives for the scalar field a low mass $m_{\phi}=44 \mathrm{GeV}$, which is of the order the Higgs one $126 \mathrm{GeV}$. In this calculation the scale $\mu$ allowing the top mass fixation is within an intermediate energy region: $\mu=11.63 \mathrm{GeV}$, which gives a coupling value $\alpha=\frac{g_{0}^{2}}{4 \pi}=0.225445$.

Therefore the mass of the scalar field waves is given by

$m_{\phi}=\sqrt{V^{\prime \prime}[\phi]}$.

That is, the mass of the scalar field in a first approximation is defined by the square root of the second derivative of the effective potential respect to the mean field. Therefore, the second derivative of the potential curve in Fig. 4, estimates for the mass of the scalar field $m_{\phi}=45 \mathrm{GeV}$. This value is smaller but of the order of the observed Higgs mass of $126 \mathrm{GeV}$. Then, after considering that by fixing the fermion mass to the top quark experimental mass, had determined a mass for the scalar field being close to the Higgs's one, directly supports the possibility of generating the breaking of symmetry in the SM through the Yukawa interaction between the Top quark and the Higgs field. This possibility is also made plausible, by noticing that upon considering a similar evaluation, but in a model showing the same field content as the SM, there will exist a variety of additional contributions to the Higgs mass. In particular it will exist a squared in the Higgs field term required by renormalization. Thus, their contribution to the Higgs potential could correct the resulting scalar field mass value to become close to the observed one.

As remarked before, the appeared single extremum of the potential is related with the existence of so called "second minimum" of the Higgs potential in the SM, laying at large values of the Higgs field. That minimum is recognized to be produced precisely by the contributions of the top-quark Yukawa interaction term, which is of the same form that the one considered here $[1,2,3]$.

\section{POTENTIAL EVALUATION USING THE RUNNING COUPLING}

In this section, we will investigate the stability of the previous evaluation of the effective potential for the scalar mean field by substituting the constant value of the coupling chosen at a 
given scale by the running coupling with momentum. The objective will be to check how robust can be the fixing of the Top quark mass, under the replacement of the constant value of the coupling by a momentum dependent one. For this purpose the expression for the finite part of the effective potential (obtained after employing the Minimal Substractions scheme) will be represented as a momentum integral, in which replacement of the constant coupling can be further implemented.

In fact this had been the most demanding technical part of the present work. The difficulty was determined by the employed dimensional regularization approach under the Minimal Substraction scheme. The obstacles were created by the fact that normally, the full divergence structure of the evaluated quantities near dimension equal to four, only appears after integrating over the momenta. But, for approximately substituting the constant coupling by the running one with momentum, it requires an expression for the finite part being represented as a momentum integral. To derive this expression is the main objective of most of the technical discussion to be presented below in this section.

The plan of the section is as a follows. First, we will present the formula for the effective potential as represented by a momentum integral in terms of Appell series as functions of the momenta and $\epsilon=\frac{4-D}{2}$. But, the Euclidean space integral has a volume differential of the form $d V=d q q^{3-2 \epsilon}$ (determined by the $D$ dimensional integration over the momenta). Then, it was noted that for making finite the integral, it is only needed to substract a specially designed asymptotic form at large values of $q$ of the factor $F(q)$ defining the full integrand as $F(q) d V$. The complex form of these terms complicated the discussion, because the Appell functions appearing do not show a pure Taylor expansion in powers of $\frac{1}{q}$. In fact, the expansion becomes a power series of $\frac{1}{q}$ with factors which are powers of $q^{\epsilon}$. These factors, although becoming equals to 1 for $\epsilon \rightarrow 0$, contribute to the final result due to the appearance of divergent pole terms in $\epsilon$.

After substracting the appropriate divergent terms, a formula for a momentum integral was obtained which became convergent at large momentum and showed a single divergent term at small momentum as $\frac{1}{q}$. However, we noted that after substituting few of these divergent factors as

$$
\frac{1}{q} \rightarrow \frac{1}{\sqrt{q^{2}+\delta^{2}}}
$$

the integral became again convergent at large momentum, but also at zero momentum. This led to the introduction of a new parameter which afterwards played a helpful role. At this point, it was possible to take the limit $\epsilon \rightarrow 0$ in the integral due to its finite character. Then, we passed to study the divergent contribution which was substracted to make the integral finite at large as well as for zero momenta. Since the momentum dependence of this substraction became simpler than the original one, it was possible to exactly evaluate the momentum integral, which allowed to determine its pole structure in $\epsilon$ as well as its finite part.
As it should result to be the case, the pole part of the divergent integral exactly reproduced the Minimal Substraction required to make the two loop quark-gluon term finite. As for its finite part, it resulted as a function of the scale parameter $\mu$, the mean field $\phi$, the strong coupling constant, but also of the new parameter $\delta$ introduced for making the substracted integral convergent at small momentum. At this point came the helpful character of the regularization parameter $\delta$ : we selected its value as a function of $\mu, \phi$ and $g$ for to impose that the finite part of the divergent integral vanishes for all values of $\mu, \phi$ and $g$. This fixation of $\delta$ became possible and a real and positive solution exists for the variety of values of $\mu, \phi$ and $g$.

Therefore, it followed that the defined finite integral over the momenta exactly coincides with the effective potential when the strong coupling is constant. Hence, the obtained formula for effective potential can be used to explore the effects of considering that the strong coupling of the gluons with the quarks runs with the exchanged momenta. This will done within the contribution associated to the quark self energy loop, contracted with the gluon propagator.

\subsection{The two loops quark-gluon contribution to the effective potential as a momentum integral}

As it was mentioned in the past subsection, we now investigate the effects that could have on the results to assume that the strong coupling varies with the magnitude of the exchanged momentum $q$. The evaluation of the effective potential done in the past section seems amenable of being influenced by assuming the coupling to run with the momentum.

We followed a specific path in order to derive a momentum integral for the finite part of the quark-gluon contribution to the effective action. The outcome, in one hand coincides with the result when the coupling is momentum independent and then it was employed to investigate the effects of substituting the strong coupling by a running with the momentum expression in the following section.

We start by considering that the effective action is given by the contraction of the gluon polarization tensor and free gluon propagator. Then, the polarization tensor was expressed through the formula derived in the page 374 of reference [5]

$$
\begin{array}{r}
\Pi_{\mu \nu}^{a b}(q)=-\frac{4 g^{2} \delta^{a b}}{(4 \pi)^{2-\epsilon}} \Gamma(\epsilon)\left(q^{2} g_{\mu \nu}-q_{\mu} q_{\nu}\right) \times \\
\int_{0}^{1} d x x(1-x)\left(\phi^{2}-x(1-x) q^{2}\right)^{-\epsilon} .
\end{array}
$$

After contracting the tensor with the gluon propagator

$D_{\mu \nu}^{a b}(q)=\frac{\delta^{a b}}{q^{2}}\left(g_{\mu \nu}-(1-\alpha) \frac{q_{\mu} q_{v}}{q^{2}}\right)$

the quark-gluon contribution to the effective action for the scalar field (minus the effective potential), after evaluating the integral over the variable $x$ got the expression 


$$
\begin{aligned}
\Gamma_{g}^{(2)}(\epsilon, g, \phi, \mu)= & \int_{0}^{\infty} d q \int_{0}^{1} d x 2^{-3+4 \epsilon} g^{2} q^{3-2 \epsilon} \pi^{-4+2 \epsilon}(-3+2 \epsilon) \times \\
& \frac{\Gamma(\epsilon)(1-x) x\left(1-q^{2}(-1+x) x\right)^{-\epsilon} \phi^{4}\left(\frac{\phi}{\mu}\right)^{-4 \epsilon}}{\Gamma(2-\epsilon)} \\
= & \int_{0}^{\infty} d q L_{g}^{(2)}(q, \epsilon, g, \phi, \mu) .
\end{aligned}
$$

where the usual momentum integration variable, let say $p$, had been expressed in terms of a dimensionless variable $q$ through the change of variables $p=\phi q$. In addition, it also was defined the momentum integrand $L_{g}^{(2)}(q, \epsilon, g, \phi, \mu)$ having the explicit form

$$
\begin{aligned}
L_{g}^{(2)}(q, \epsilon, g, \phi, \mu)= & \frac{1}{3 \Gamma(2-\epsilon)} g^{2} q^{3-2 \epsilon} \times \\
& 4^{\epsilon}(2 \pi)^{-4+2 \epsilon}(3-2 \epsilon) \phi^{4}\left(\frac{\phi}{\mu}\right)^{-4 \epsilon} \Gamma(\epsilon) \times \\
& \left(-3 \operatorname{AppelF}_{1}[2, \epsilon, \epsilon, 3, u(q), v(q)]+\right. \\
& -2 \operatorname{AppelF}_{1}[3, \epsilon, \epsilon, 4, u(q), v(q)], \\
u(q)= & \frac{\left(-q^{2}+\sqrt{q^{2}\left(4+q^{2}\right)}\right)}{2}, \\
v(q)= & \frac{\left(-q^{2}-\sqrt{q^{2}\left(4+q^{2}\right)}\right)}{2},
\end{aligned}
$$

in terms of the Appell functions $[8,9]$

$$
\begin{gathered}
\operatorname{AppelF}_{1}\left[a, b_{1}, b_{2}, c, x, y\right]=\sum_{m=0}^{\infty} \sum_{n=0}^{\infty} \frac{(a)_{m+n}\left(b_{1}\right)_{m}\left(b_{2}\right)_{n}}{m ! n !(c)_{m+n}} \times \\
x^{m} y^{n},
\end{gathered}
$$

in which the Pochhammer symbols are defined as

$(a)_{n}=\frac{\Gamma(a+n)}{\Gamma(a)}$.
The $L_{g}^{(2)}$ function is not convergent at large momentum, and the pole parts of its momentum integral as functions of the $\epsilon$ parameter define the Minimal Substraction required to make the result finite. However, as it was mentioned before, by substracting the asymptotic behavior of the Appell functions at large momentum the integral can be made finite. The resulting integrand after this substraction can be written as

$$
\begin{aligned}
& L_{s u b}(q, \epsilon, g, \phi, \mu, \delta)=\frac{1}{3 \Gamma(2-\epsilon)} g^{2} q^{3-2 \epsilon} \times \\
& 4^{\epsilon}(2 \pi)^{-4+2 \epsilon}(3-2 \epsilon) \phi^{4}\left(\frac{\phi}{\mu}\right)^{-4 \epsilon} \Gamma(\epsilon) \times \\
& \text { (-3 } \text { AppelF }_{1}[2, \epsilon, \epsilon, 3, u(q), v(q)] \\
& \text { - Appel23Sub }[q, \epsilon, \delta])+ \\
& 2\left(\text { AppelF }_{1}[3, \epsilon, \epsilon, 4, u(q), v(q)]-\right. \\
& \text { Appel34Sub }[q, \epsilon, \delta])]
\end{aligned}
$$

where the substractions done are defined by the large momentum asymptotic form of the two entering Appell functions given by the fomulae 


$$
\begin{aligned}
& \operatorname{Appel} 23 \operatorname{Sub}[q, \epsilon, \delta]=\frac{2^{-1+2 \epsilon} \pi^{\frac{3}{2}} q^{-2 \epsilon} \csc (\pi(1-\epsilon))}{\Gamma\left(\frac{3}{2}-\epsilon\right) \Gamma(\epsilon)}+ \\
& \left(\frac{1}{q^{2}}\right)^{1-\epsilon} q^{-2 \epsilon}\left(\frac{2^{1+2 \epsilon} \pi^{\frac{3}{2}} q^{-2 \epsilon} \epsilon \csc (\pi(1-\epsilon))}{\Gamma\left(\frac{3}{2}-\epsilon\right) \Gamma(\epsilon)}-\right. \\
& \left.\frac{2 \pi \csc (\pi(1-\epsilon))}{\Gamma(2-\epsilon) \Gamma(\epsilon)}+\frac{4^{\epsilon} \pi^{\frac{3}{2}} q^{-2 \epsilon} \epsilon \csc (\pi(1-\epsilon))}{\Gamma\left(\frac{3}{2}-\epsilon\right) \Gamma(1+\epsilon)}\right)+ \\
& \left(\frac{1}{q^{2}}\right)^{-\epsilon} \frac{q^{-2 \epsilon}}{\left(q^{2}+\delta^{2}\right)}\left(\frac{4^{1+\epsilon} \pi^{\frac{3}{2}} q^{-2 \epsilon} \epsilon \csc (\pi(1-\epsilon))}{\Gamma\left(\frac{3}{2}-\epsilon\right) \Gamma(\epsilon)}+\right. \\
& \frac{4^{1+\epsilon} \pi^{\frac{3}{2}} q^{-2 \epsilon} \epsilon^{2} \csc (\pi(1-\epsilon))}{\Gamma\left(\frac{3}{2}-\epsilon\right) \Gamma(\epsilon)}+\frac{8 \pi \csc (\pi(1-\epsilon))}{\Gamma(2-\epsilon) \Gamma(\epsilon)}- \\
& \frac{12 \pi \csc (\pi(1-\epsilon))}{\Gamma(3-\epsilon) \Gamma(\epsilon)}+\frac{8 \pi \epsilon \csc (\pi(1-\epsilon))}{\Gamma(3-\epsilon) \Gamma(\epsilon)}- \\
& \frac{4^{1+\epsilon} \pi^{\frac{3}{2}} q^{-2 \epsilon} \epsilon \csc (\pi(1-\epsilon))}{\Gamma\left(\frac{3}{2}-\epsilon\right) \Gamma(1+\epsilon)}- \\
& \frac{4^{1+\epsilon} \pi^{\frac{3}{2}} q^{-2 \epsilon} \epsilon^{2} \csc (\pi(1-\epsilon))}{\Gamma\left(\frac{3}{2}-\epsilon\right) \Gamma(1+\epsilon)}+ \\
& \frac{3 \times 4^{\epsilon} \pi^{\frac{3}{2}} q^{-2 \epsilon} \epsilon \csc (\pi(1-\epsilon))}{\Gamma\left(\frac{3}{2}-\epsilon\right) \Gamma(2+\epsilon)}+ \\
& \left.\frac{3 \times 4^{\epsilon} \pi^{\frac{3}{2}} q^{-2 \epsilon} \epsilon^{2} \csc (\pi(1-\epsilon))}{\Gamma\left(\frac{3}{2}-\epsilon\right) \Gamma(2+\epsilon)}\right),
\end{aligned}
$$

and

$$
\begin{aligned}
\operatorname{Appel34Sub}[q, \epsilon, \delta] & =\frac{3}{2 q^{2}(1-\epsilon)}+\frac{3}{4} \operatorname{Appel} 23 \operatorname{Sub}[q, \epsilon, \delta]- \\
& \frac{3 \times 2^{-4+2 \epsilon} \pi^{\frac{3}{2}} q^{-2 \epsilon} \epsilon^{2} \csc (\pi(2-\epsilon))}{(-1+\epsilon) \Gamma\left(\frac{5}{2}-\epsilon\right) \Gamma(-1+\epsilon)}+ \\
& 2^{-2 \epsilon}\left(\frac{1}{q^{2}}\right)^{1-\epsilon}\left(\frac{3 \times 2^{-2+4 \epsilon} \pi^{\frac{3}{2}} q^{-2 \epsilon} \csc (\pi(2-\epsilon))}{\Gamma\left(\frac{5}{2}-\epsilon\right) \Gamma(-1+\epsilon)}-\right. \\
& \left.\frac{3 \times 2^{-3+4 \epsilon} \pi^{\frac{3}{2}} q^{-4 \epsilon} \csc (\pi(2-\epsilon))}{\Gamma\left(\frac{5}{2}-\epsilon\right) \Gamma(\epsilon)}\right) \\
& \frac{2^{-2 \epsilon} q^{2 \epsilon}}{\left(q^{2}+\delta^{2}\right)}\left(-\frac{3 \times 2^{-1+4 \epsilon} \pi^{\frac{3}{2}} q^{-4 \epsilon} \epsilon \csc (\pi(2-\epsilon))}{\Gamma\left(\frac{5}{2}-\epsilon\right) \Gamma(-1+\epsilon)}+\right. \\
& \frac{3 \times 2^{2 \epsilon} \pi q^{-2 \epsilon} \csc (\pi(2-\epsilon))}{(-1+\epsilon) \Gamma(3-\epsilon) \Gamma(-1+\epsilon)}+ \\
& \frac{3 \times 2^{-1+4 \epsilon} \pi^{\frac{3}{2}} q^{-4 \epsilon} \epsilon \csc (\pi(2-\epsilon))}{\Gamma\left(\frac{5}{2}-\epsilon\right) \Gamma(\epsilon)}- \\
& \left.+\frac{9 \times 2^{-3+4 \epsilon} \pi^{\frac{3}{2}} q^{-4 \epsilon} \epsilon \csc (\pi(2-\epsilon))}{\Gamma\left(\frac{5}{2}-\epsilon\right) \Gamma(1+\epsilon)}\right)
\end{aligned}
$$


In the above two expressions, it should be noted that earlier defined quantity $\delta$ is appearing in the denominators of the form $\left(q^{2}+\delta^{2}\right)$. They, appeared after making the substitution $q \rightarrow \frac{1}{\sqrt{q^{2}+\delta^{2}}}$ in a $q^{4}$ denominator of the only term diverging as $\frac{1}{q}$ at zero momentum. This procedure eliminates the mentioned zero momentum divergence, furnishes a simple momentum dependence of the result and makes the result a function of the quantity $\delta$. The substitution, on another hand does not disturbe the large momentum convergence of the considered integral. Therefore, the substracted divergent expression has the form

$$
\begin{aligned}
& L_{\text {count }}(q, \epsilon, g, \phi, \mu, \delta)=\frac{1}{3 \Gamma(2-\epsilon)} g^{2} q^{3-2 \epsilon} \times \\
& 4^{\epsilon}(2 \pi)^{-4+2 \epsilon}(3-2 \epsilon) \Gamma(\epsilon) \phi^{4}\left(\frac{\phi}{\mu}\right)^{-4 \epsilon} \times \\
&(-3 \operatorname{Appel} 23 \operatorname{Sub}(q, \epsilon, \delta)+ \\
&\quad 2 \operatorname{Appel34Sub}(q, \epsilon, \delta)) .
\end{aligned}
$$

Now, as remarked above, the relative simplicity of the obtained momentum dependence of the substracted term, allows to exactly perform the momentum integrals to obtain the result

$$
\begin{aligned}
& S_{\text {count }}(\epsilon, g, \phi, \mu, \delta)=\int_{0}^{\infty} d q L_{\text {count }}(q, \epsilon, g, \phi, \mu, \delta) \\
& =4^{-5+3 \epsilon} g^{2} \pi^{-\frac{3}{2}+2 \epsilon}\left(\frac{1}{\delta^{2}}\right)^{\epsilon} \times \\
& (-3+2 \epsilon) \phi^{4}\left(\frac{\phi}{\mu}\right)^{-4 \epsilon} \csc (\pi \epsilon)^{2} \times \\
& \left(-4^{2+\epsilon} \pi^{\frac{3}{2}}\left(\frac{1}{\delta^{2}}\right)^{\epsilon}(-2+\epsilon)(-1+\epsilon)^{2} \times\right. \\
& \epsilon(-1+2 \epsilon) \csc (2 \pi \epsilon) \Gamma(2-2 \epsilon) \Gamma(1+\epsilon)- \\
& 2^{1+2 \epsilon} \pi^{\frac{3}{2}}\left(\frac{1}{\delta^{2}}\right)^{\epsilon}(-2+\epsilon)(-1+2 \epsilon) \times \\
& (-3+4 \epsilon) \csc (2 \pi \epsilon) \Gamma(3-2 \epsilon) \Gamma(1+\epsilon)- \\
& 4^{\epsilon} \pi^{\frac{3}{2}}\left(\frac{1}{\delta^{2}}\right)^{\epsilon}(-1+2 \epsilon)(1+4 \epsilon) \times \\
& \csc (2 \pi \epsilon) \Gamma(5-2 \epsilon) \Gamma(1+\epsilon)- \\
& 64(-2+\epsilon)(-1+\epsilon)^{2} \Gamma(2-2 \epsilon) \times \\
& \Gamma\left(\frac{5}{2}-\epsilon\right) \Gamma(-1+\epsilon) \Gamma(1+\epsilon)+ \\
& 64(-1+\epsilon)^{3} \Gamma(2-2 \epsilon) \Gamma\left(\frac{5}{2}-\epsilon\right) \times \\
& \Gamma(-1+\epsilon) \Gamma(1+\epsilon)+ \\
& 4^{1+\epsilon} \pi^{\frac{3}{2}}\left(\frac{1}{\delta^{2}}\right)^{\epsilon} \epsilon(-1+2 \epsilon) \csc (2 \pi \epsilon) \times \\
& \Gamma(5-2 \epsilon) \Gamma(2+\epsilon)) / \\
& \left(\Gamma\left(\frac{3}{2}-\epsilon\right) \Gamma(2-2 \epsilon)^{2} \Gamma\left(\frac{5}{2}-\epsilon\right) \times\right. \\
& \Gamma(3-\epsilon) \Gamma(-1+\epsilon) \Gamma(1+\epsilon)) \text {. }
\end{aligned}
$$

But, expanding this relation in series of the $\epsilon$ parameter leads to the result

$$
\begin{aligned}
S_{\text {count }}(\epsilon, g, \phi, \mu, \delta) & =\sum_{n=-\infty}^{\infty} S_{\text {count }}^{(n)}(g, \phi, \mu, \delta) \epsilon^{n} \\
& =-\frac{3 g^{2} \phi^{4}}{32 \pi^{4} \epsilon^{2}}+\frac{g^{2} \phi^{4}}{32 \pi^{4} \epsilon} \times \\
& \left(-7+6 \gamma-6 \log (4 \pi)+12 \log \left(\frac{\phi}{\mu}\right)\right)+ \\
& \frac{g^{2} \phi^{4}}{64 \pi^{4}} \times\left(-21+\pi^{2}+10 \gamma-\right. \\
& 48 \log (2)^{2}-28 \log (4 \pi)+ \\
& 6\left(\gamma^{2}+\gamma(3-2 \gamma)-\log (\pi) \log \left(256 \pi^{2}\right)+\right. \\
& \left.\gamma\left(-\gamma+\log \left(256 \pi^{4}\right)\right)\right)- \\
& 18 \log \left(\frac{1}{\delta^{2}}\right)+6 \log \left(\frac{1}{\delta^{2}}\right)^{2}+ \\
& \left.8\left(7-6 \gamma+6 \log (4 \pi)-6 \log \left(\frac{\phi}{\mu}\right)\right) \log \left(\frac{\phi}{\mu}\right)\right) \\
& +O^{(1)}(\epsilon),
\end{aligned}
$$

where $O^{(1)}(\epsilon)$ is a function vanishing when $\epsilon \rightarrow 0$.

In the above formula, it should be remarked that the pole part, which defines the divergent contribution, exactly coincides with the Minimal Substraction term (29) required to make finite the quark-gluon cntribution to the effective action. Now, it can be noticed that the expression for the integral $S_{\text {count }}(\epsilon, g, \phi, \mu, \delta)$ (which was substracted from the momentum integral of the term $L(q, \epsilon, g, \phi, \mu)$ to obtain a finite remaining integral) has a finite part when $\epsilon \rightarrow 0$. But, this finite part is depending on the regularization parameter $\delta$ which was used to make convergent the momentum integral around the zero momentum. This circumstance opens the interesting possibility of choosing this value of $\delta$ precisely to force the finite part to vanish for all the value of the scale $\mu$ and the mean field. Then, imposing this condition for determining $\delta$, we may write

$$
\begin{aligned}
& 0=\frac{g^{2} \phi^{4}}{64 \pi^{4}} \times(-21+\pi^{2}+10 \gamma- \\
& 48 \log (2)^{2}-28 \log (4 \pi)+ \\
& 6\left(\gamma^{2}+\gamma(3-2 \gamma)-\log (\pi) \log \left(256 \pi^{2}\right)+\right. \\
&\left.\gamma\left(-\gamma+\log \left(256 \pi^{4}\right)\right)\right)- \\
& 18 \log \left(\frac{1}{\delta^{2}}\right)+6 \log \left(\frac{1}{\delta^{2}}\right)^{2}+ \\
&\left.8\left(7-6 \gamma+6 \log (4 \pi)-6 \log \left(\frac{\phi}{\mu}\right)\right) \log \left(\frac{\phi}{\mu}\right)\right) .
\end{aligned}
$$

One helpful property of this equation is the fact that it does not involve the values of the strong coupling. This means that $\delta$ is only a function of the scale $\mu$ and the mean field $\phi$. The equation for $\delta$ has a real and positive solution defined for all 
the values of the ratio $\frac{\phi}{\mu}$ which can be expressed as follows

$$
\begin{aligned}
\delta(\phi, \mu) & =\frac{1}{\sqrt{\exp \left(f_{1}(36+4 \mathcal{F}(\phi, \mu))\right.}}, \\
\mathcal{F}(\phi, \mu) & =\sqrt{f_{2}+f_{3} \log \left(\frac{\phi}{\mu}\right)+f_{4} \log \left(\frac{\phi}{\mu}\right)^{2}}, \\
f_{1} & =0.041666 \\
f_{2} & =750.872709 \\
f_{3} & =-898.696871 \\
f_{4} & =+288.00 .
\end{aligned}
$$

The dependence of $\delta$ of the ratio $\frac{\phi}{\mu}$ is depicted in Fig. 5 .

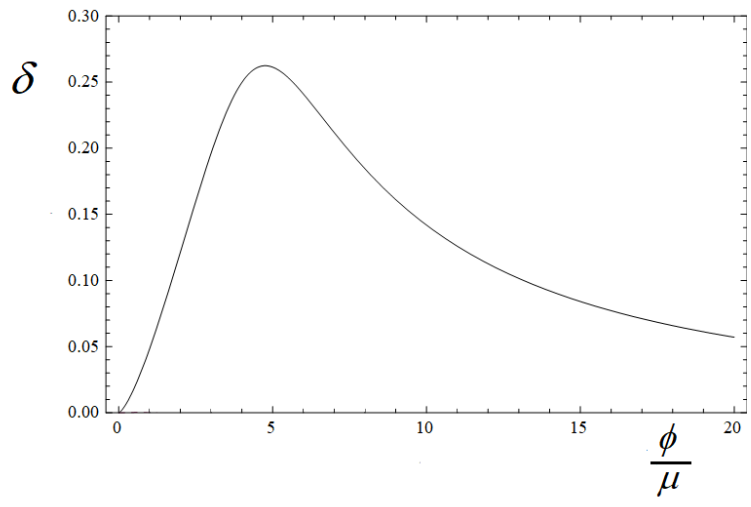

FIGURE 5: It shows the plot of the real and positive solution for $\delta(\phi, \mu)$ as a function of the ratio $\frac{\phi}{\mu}$.

Further, the function $\delta(\phi, \mu)$ was substituted in the integrand $L_{s u b}$ defined in equation (52) which upon integration furnishes the finite integral. The result for the integrand of the finite integral can then be written in the form, where a superindex of the form $\left(n_{1}, n_{2}, n_{3}, n_{4}, n_{5}, n_{6}\right)$ in the appearing Appell functions represents the numbers of $n_{i}, i=$ $1, \ldots, 6$ of the derivatives over the corresponding six arguments of such functions. It should be noticed that in writing this expression, the limit $\epsilon \rightarrow 0$ was also chosen, as allowed by the finiteness of the integral. Therefore, we have arrived to an expression of the finite part of the effective action in the form

$\mathcal{L}(g, \phi, \mu)=\int d q L_{M S}(q, g, \phi, \mu)$.

Figure 6 shows the evaluations of the quark-gluon contribution of the effective potential (minus the action) through both formulae (28) and (67). The plots for constant coupling

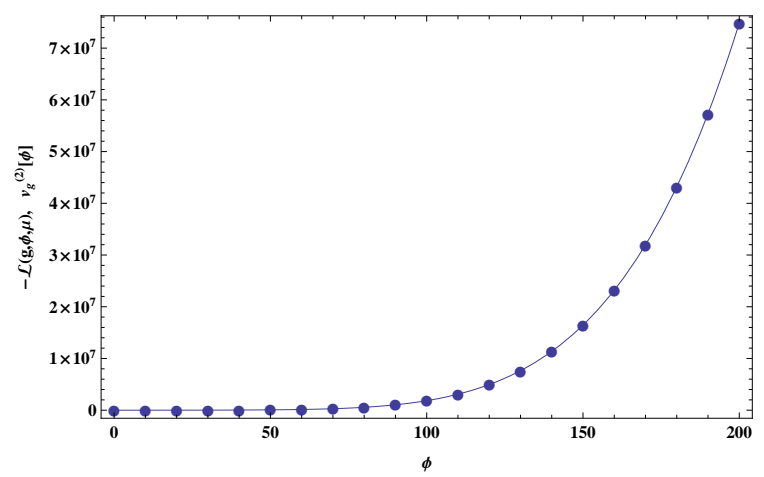

FIGURE 6: The plots illustrate the coincidence of the calculated momentum integral representation for the quark-gluon two loop contribution to the effective action with the result of the direct evaluation of the same quantity. The solid curve indicates the values of the momentum integral representation in (67) and the dotted one the evaluation of the two loop quark gluon contribution in formula (28).

$$
\begin{aligned}
L_{M S}(q, g, \phi, \mu) & =\left.L_{s u b}(q, \epsilon, g, \phi, \mu, \delta)\right|_{\delta \rightarrow \delta(\phi, \mu), \epsilon \rightarrow 0} \\
& =\frac{g^{2} \phi^{4}}{32 \pi^{4} q}\left(12.9266-12 q^{2}+2.1789 q^{4}-12 \gamma+2 \gamma q^{4}+\right. \\
& +\frac{0.643577-0.597445 \gamma-0.597445 \log \left(\frac{1}{q^{2}}\right)}{\left(0.22313+\exp \left(0.166667 \sqrt{750.873+\log \left(\frac{\phi}{\mu}\right)\left(-898.697+288 \log \left(\frac{\phi}{\mu}\right)\right)}\right) q^{2}\right)^{2}}- \\
& 12 \log \left(\frac{1}{q^{2}}\right)+6 q^{2} \log \left(\frac{1}{q^{2}}\right)+ \\
& \frac{-22313+\exp \left(0.166667 \sqrt{750.873+\log \left(\frac{\phi}{\mu}\right)\left(-898.697+288 \log \left(\frac{\phi}{\mu}\right)\right)}\right) q^{2}}{}+ \\
& 12 q^{2} \log (q)-4 q^{4} \log (q)+ \\
& \left.q^{4}\left(-6 \operatorname{AppellF}_{1}^{(0,0,1,0,0,0)}\left(2,0,0,3, \frac{1}{2}\left(-q^{2}+\sqrt{q^{2}\left(4+q^{2}\right.}\right)\right), \frac{1}{2}\left(-q^{2}-\sqrt{q^{2}\left(4+q^{2}\right.}\right)\right)\right)+ \\
& 4 \operatorname{AppellF}_{1}^{(0,0,1,0,0,0)}\left(3,0,0,4, \frac{1}{2}\left(-q^{2}+\sqrt{q^{2}\left(4+q^{2}\right)}\right), \frac{1}{2}\left(-q^{2}-\sqrt{q^{2}\left(4+q^{2}\right)}\right)\right)- \\
& -6 \operatorname{AppellF}_{1}^{(0,1,0,0,0,0)}\left(2,0,0,3, \frac{1}{2}\left(-q^{2}+\sqrt{q^{2}\left(4+q^{2}\right)}\right), \frac{1}{2}\left(-q^{2}-\sqrt{q^{2}\left(4+q^{2}\right)}\right)\right)+ \\
& \left.\left.4 \operatorname{AppellF}_{1}^{(0,1,0,0,0,0)}\left(3,0,0,4, \frac{1}{2}\left(-q^{2}+\sqrt{q^{2}\left(4+q^{2}\right)}\right), \frac{1}{2}\left(-q^{2}-\sqrt{q^{2}\left(4+q^{2}\right)}\right)\right)\right)\right),
\end{aligned}
$$


through are done for values of the scale parameter $\mu=11.63$ $\mathrm{GeV}$ and of the coupling $g=g_{o}(11.63, \Lambda)$. The solid curve shows the values of $-\mathcal{L}(g, \phi, \mu)$ and the dotted one the values of $v_{g}^{(2)}[\phi]$ in (28) as functions of the mean field.

In expression (67), it is possible now to replace the up to now constant value of the strong coupling by its running expression with momentum. The following section will discuss some properties of this substitution.

\section{THE USE OF THE RUNNING COUPLING WITH MOMENTUM}

In this section, the discussion starts by defining the running coupling to be considered. Since it is known that the values of couplings are not well defined at low momenta of the order of 1 $\mathrm{GeV}$, we defined values of the running coupling "saturated " at low momentum. That is, in a neighborhood of zero momentum they were assumed to be constant.

For the purpose of substituting the constant coupling by its running counterpart, we analyzed two variants of couplings. The first of them was the expression for the one loop renormalization coupling as a function of momentum

$g_{o}(q)=\left\{\begin{array}{cc}\sqrt{\frac{1}{b_{o} \log \left(\frac{\phi^{2} q^{2}}{\Lambda^{2}}\right)},}, \frac{\phi^{2} q^{2}}{\Lambda^{2}}>\exp \left(\frac{16 \pi^{2}}{7 g_{\text {sat }}^{2}}\right) \\ g_{\text {sat }}, \quad \frac{\phi^{2} q^{2}}{\Lambda^{2}}<\exp \left(\frac{16 \pi^{2}}{7 g_{\text {sat }}^{2}}\right)\end{array}\right.$,

$g_{\text {sat }}=2.06$.

Note that the usual momentum argument of the running coupling had been substituted in terms of the previously defined dimensionless integration variable $q$. In this expression the couplings for momenta smaller than the value at which they become equal to the highest measured coupling $g_{\text {sat }}=2.06$, are assumed to remain constant, and equal to their "saturation" values [10]. Another form of the analyzed running coupling was given by an interpolation of the set of experimental values reported in reference [10]. The expression describing the data was obtained in the form

$g_{\exp }(q)=\left\{\begin{array}{cc}\frac{23.4193}{\log \left(32361.1672(\phi q)^{2}\right)}, & \phi q>1.604 \\ g_{\text {sat }}, & \phi q<1.604\end{array}\right.$,

where $g_{\text {sat }}$ is the same saturation value defined before, that is, the maximal value of the experimentally measured couplings given in reference [10]. Note that the dimensional momentum momentum $p$ entering the defintion of the experimental values of the couplings was expressed in terms of the before defined dimensionaless integration variable $q$ through $p=\phi q$.

Both couplings momentum behavior are plotted in Fig. 7 as functions of the momentum $\phi q$. As it can noticed the values of the observations are systematically smaller than the one loop determined values. Therefore, in what follows we decided to employ the fitting curve of the experimental values for substituting the constant coupling in formula (67).

\subsection{The quark-gluon effective potential evaluation using the running coupling}

It is possible now to substitute the expression for the experimental value of the running coupling $g_{\exp }(q)$ in (67) to define the quark-gluon contribution to the effective potential as evaluated at the running coupling values, in the form

$V_{\text {run }}(\phi, \mu)=-\left.\int d q L_{M S}(q, g, \phi, \mu)\right|_{g \rightarrow g_{\exp }(q)}$.

The resulting formula for this contribution to the effective potential takes the form

$$
\begin{aligned}
V_{\text {run }}(\phi, \mu) & =\int_{0}^{\infty} d q \frac{g_{\exp }(q)^{2} \phi^{4}}{32 \pi^{4} q}\left(12.9266-12 q^{2}+2.1789 q^{4}-12 \gamma+2 \gamma q^{4}+\right. \\
& +\frac{0.643577-0.597445 \gamma-0.597445 \log \left(\frac{1}{q^{2}}\right)}{\left(0.22313+\exp \left(0.166667 \sqrt{750.873+\log \left(\frac{\phi}{\mu}\right)\left(-898.697+288 \log \left(\frac{\phi}{\mu}\right)\right)}\right) q^{2}\right)^{2}}- \\
& 12 \log \left(\frac{1}{q^{2}}\right)+6 q^{2} \log \left(\frac{1}{q^{2}}\right)+ \\
& -5.76862+5.35512 \gamma+5.35512 \log \left(\frac{1}{q^{2}}\right) \\
& 12 q^{2} \log (q)-4 q^{4} \log (q)+ \\
& q^{4}\left(-6 \operatorname{AppellF}_{1}^{(0,0,1,0,0,0)}\left(2,0,0,3, \frac{1}{2}\left(-q^{2}+\sqrt{q^{2}\left(4+q^{2}\right)}\right), \frac{1}{2}\left(-q^{2}-\sqrt{q^{2}(4}+q^{2}\right)\right)\right)+ \\
& 4 \operatorname{AppellF}_{1}^{(0,0,1,0,0,0)}\left(3,0,0,4, \frac{1}{2}\left(-q^{2}+\sqrt{q^{2}\left(4+q^{2}\right)}\right), \frac{1}{2}\left(-q^{2}-\sqrt{q^{2}\left(4+q^{2}\right)}\right)\right)- \\
& \left.-6 \operatorname{AppellF}_{1}^{(0,1,0,0,0,0)}\left(2,0,0,3, \frac{1}{2}\left(-q^{2}+\sqrt{q^{2}\left(4+q^{2}\right.}\right)\right), \frac{1}{2}\left(-q^{2}-\sqrt{q^{2}\left(4+q^{2}\right.}\right)\right)+ \\
& \left.\left.4 \operatorname{AppellF}_{1}^{(0,1,0,0,0,0)}\left(3,0,0,4, \frac{1}{2}\left(-q^{2}+\sqrt{q^{2}\left(4+q^{2}\right)}\right), \frac{1}{2}\left(-q^{2}-\sqrt{q^{2}\left(4+q^{2}\right)}\right)\right)\right)\right) .
\end{aligned}
$$

Next, it was possible to evaluate the effects of the running on the calculation of the effective potential. It can be recalled 


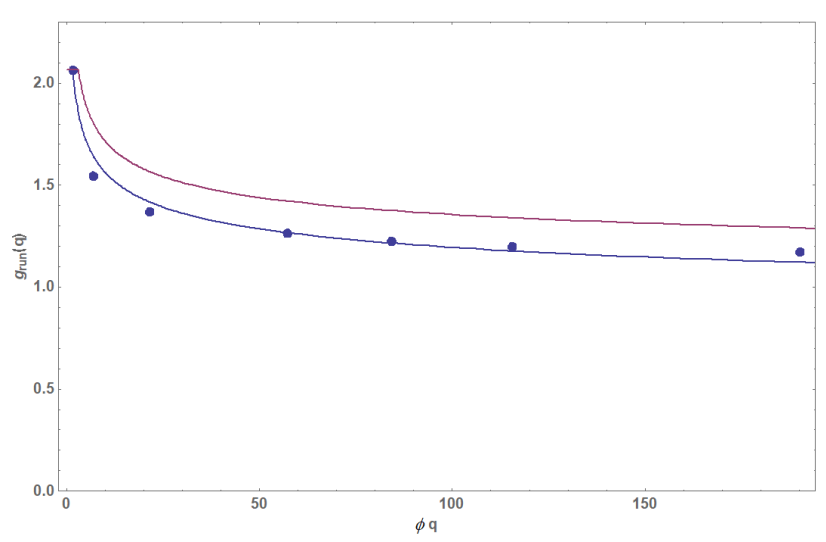

FIGURE 7: It shows the values of the fitting formula for a number of experimentally measured values of the strong coupling according to reference [10]. This curve is the lower one and the fitted experimental points are indicated by the dots. The higher plot shows the values of the one loop renormalization group running coupling.

that before, we have been able to fix the Top quark mass by fixing the minimum of the potential (after calculated at constant strong coupling) at the scale parameter value $\mu=11.63 \mathrm{GeV}$. We then firstly calculated $V_{\text {run }}(\phi, \mu)$ at this scale. The evaluation of the total effective potential was done by using the formula

$V_{\text {run }}^{\text {total }}(\phi, \mu)=v^{(1)}[\phi, \mu]+v_{Y}^{(2)}[\phi, \mu]+V_{\text {run }}(\phi, \mu)$,

in which the quark-gluon term is calculated using the above formula for $V_{\text {run }}(\phi, \mu)$. The one loop and scalar two loop contributions were defined by the same formulae (15) and (33) which were used in calculating the potential for constant coupling, since they do no depend on the running coupling. But, for the original value of the scale $\mu=11.63 \mathrm{GeV}$, the potential curve was appreciably modified. By example, the minimum position was changed with respect to the mean field determining a Top mass value near $175 \mathrm{GeV}$.

However, through a reduction of the value of $\mu$ from 11.63 to $4.95 \mathrm{GeV}$, it was possible to maintain the minimum of the potential at the $175 \mathrm{GeV}$ value for the mass of the quark. The results are plotted in Fig. 8. The solid curve represents the values of the potential evaluated by using the running coupling evaluated at a smaller value for the scale $\mu=4.95 \mathrm{GeV}$. The dashed one is the potential values previously calculated by employing constant coupling values, chosen at $\mu=11.63 \mathrm{GeV}$. Therefore, it resulted in the spontaneous symmetry braking pattern obtained at constant coupling was not drastically affected by the consideration that the coupling varies with the momentum scale.

Next, after evaluating the mass of the scalar field the result was close to the value associated to a constant coupling

$$
\begin{aligned}
m_{\phi}^{\text {run }} & =\sqrt{V_{\text {run }}^{\text {total }} " \prime[0]} \\
& =53.58 \mathrm{GeV} .
\end{aligned}
$$

It should be remarked that the scalar field mass values obtained here are smaller than the observed Higgs particle mass

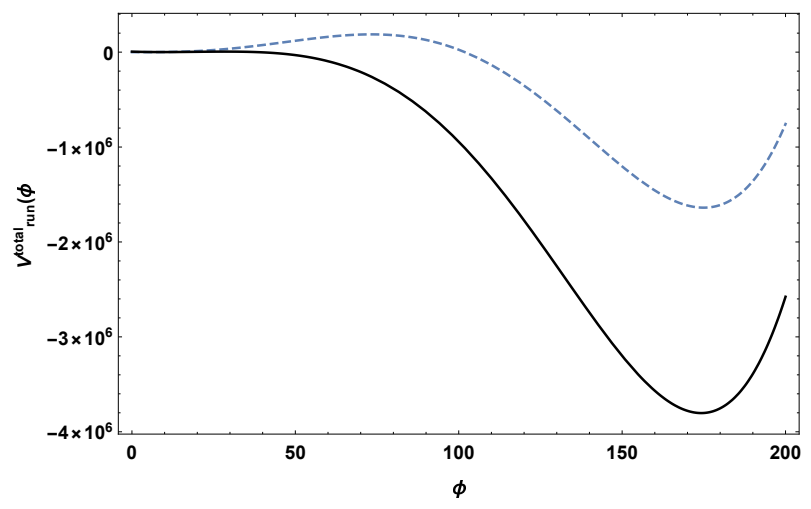

FIGURE 8: It shows the plot of the values of the total two-loop effective potential for the scalar field (solid curve) after the running coupling is employed for calculating the quark-gluon contribution. The dashed curve shows the similar potential as evaluated for the constant values of the strong coupling. The results indicate that the momentum dependence of the coupling affects the values of the effective potential. However, the running coupling does not drastically disturb the arising spontaneous symmetry breaking pattern, by allowing to again fix the minimum of the potential at the scalar field value furnishing the Top quark mass. For this purpose, it was only required to reduce the scale from $\mu=11.63$ down to $\mu=4.95 \mathrm{GeV}$.

of $126 \mathrm{GeV}$. After thinking about this outcome, we consider that it does not represent a direct negative result in connection of the studied possibility of basing the SM on a symmetry breaking associated to the Yukawa Top-Higgs interaction. This conclusion is determined by the following reasoning. If we consider a similar calculation of the Higgs mass in the framework of the more complex SM, then, there will be various new contributions to the Higgs mass. In particular, the action of the model should include a quadratic in the Higgs field term, as required by renormalization (as well also a quartic in the field term). This term by itself can allow to fix the observed value of the Higgs. Evenmore, the small result for the Higgs mass arising is helpful in this sense, since it allows the new term to be a positive mass squared one. Therefore, one important outcome of the present work is to determine that the spontaneous symmetry breaking generated by a single quark and a scalar (upon fixing the observed quark top mass) produces a mass for the scalar field being smaller than the Higgs one. We estimate that the results of the present work suggest the interest of attempting to construct a modified SM upon the here investigated spontaneous symmetry effect.

\section{CONNECTIONS BETWEEN THE SIMPLE MODEL AND THE SM}

In this final section, we comment about the motivation to examining the simple theory discussed here. The model considers only two scalar and fermion fields lacking the SU(2) structure of the Higgs particle and the quarks. We have chosen this simplification in an attempt to isolate the problematic of the so called "second minimum" in the SM. This extremum of the Higgs potential is known to be mainly determined by the interactions between only two fields: the Higgs and the Top quark ones. Therefore, we decided to include in the model only two 
simple scalar and fermion fields resembling the Higgs and the Top modes. The fact that after the breaking of the $\mathrm{SU}(2) \times \mathrm{U}(1)$ symmetry the three goldstone bosons become unphysical, supports this expectation.

One important question consists in whether or not the obtained results support that analogous conclusions could be attained from a model being almost identical to the SM, but only excluding the Mexican Hat Higgs potential. For analyzing this point, let us review each of the basic contributions to the potential. A main one is the one loop quark term which is logarithmic of the form

$\frac{V_{1}(\phi)}{\phi^{4}}=-v_{1} \log \left(\frac{\phi}{\mu}\right)$,

in which $v_{1}$ is a positive number. This term is mainly responsible of the existence of the "second minimum" in the SM. This is because it is unbounded from below, which can define a radiative correction minimum, if larger than $\log \left(\frac{\phi}{\mu}\right)$ positive components of the potential for large mean fields exist. Such large potentials as functions of $\phi$ in the model are furnished by the two loops terms determined by the gluons and the scalar. The main in this example, gluon potential has the leading behavior

$\frac{V_{2}(\phi)}{\phi^{4}} \sim v_{2} g^{2}\left(\log \left(\frac{\phi}{\mu}\right)\right)^{2}$,

in which $v_{2}$ is positive. For constant coupling $\alpha=0.225$ the Top quark mass for the fermion was possible to be fixed by choosing $\mu=11.63 \mathrm{GeV}$, which determined a coupling of $\alpha=0.225$. Since the experimental values of the running coupling (as a function of the momenta) are in general smaller than the one loop ones (as a function of the scale $\mu$ chosen as equal to the momentum) the two loop gluon contribution becomes smaller when it is evaluated using the running coupling. Thus, since the leading two loop terms are quadratic in $\log \left(\frac{\phi}{\mu}\right)$, it is required to decrease the value of $\mu=11.63 \mathrm{GeV}$, in order to retain the minimum in the same position of the scalar field assuring the Top quark mass for the fermion. Note that a change of $\mu$ by a new value $\mu^{\prime}$ in the one loop term only adds a constant independent of $\phi$ term $\log \left(\frac{\mu}{\mu^{\prime}}\right)$ to the function $\frac{V_{1}(\phi)}{\phi^{4}}$. Then, it does not modify the minimum position because the extremum follows from the vanishing of the derivative of the potential divided by $\phi^{4}$.

Now, let us consider that a similar analysis will be done for a modified SM in which the Higgs potential is chosen to have the form

$V_{\text {Higgs }}(\phi)=\frac{m^{2}}{2} \phi^{2}+\frac{\lambda}{4 !} \phi^{4}$,

in which the mass squared term is positive in place of the usual negative one generating the Mexican Hat potential. It can be observed that the one loop fermion contribution will have a similar form (with the sole expected modification of a factor two due to the isotopic SU(2) components of the Top quark)

$V_{1}^{S M}(\phi)=-v_{1}^{S M} \log \left(\frac{\phi}{\mu}\right) \phi^{4}$

But, for the various two loops contributions associated to this model (like the massive $W, Z$ and the Higgs field) the lead- ing high $\phi$ behavior can be expected to have a form of the type

$$
\begin{gathered}
V_{2}^{S M}(\phi) \sim\left(v_{2}^{g} g(\mu)^{2}+v_{2}^{e} g_{e}(\mu)^{2}+\right. \\
\left.v_{2}^{w} g_{w}(\mu)^{2}+\ldots\right) \times\left(\log \left(\frac{\phi}{\mu}\right)\right)^{2} \phi^{4},
\end{gathered}
$$

in which the appearing couplings should be the strong, the electromagnetic and the weak ones. Adding the three types of contributions the total potential should take the generic form

$$
\begin{aligned}
V_{T}^{S M}(\phi) & \sim \frac{m^{2}}{2} \phi^{2}+\frac{\lambda}{4 !} \phi^{4}-v_{1}^{S M} \log \left(\frac{\phi}{\mu}\right) \phi^{4}+ \\
& \left(v_{2}^{g} g^{2}(\mu)+v_{2}^{W} g_{W}^{2}(\mu)+\right. \\
& \left.v_{2}^{Z} g_{Z}^{2}(\mu)+\ldots\right)\left(\log \left(\frac{\phi}{\mu}\right)\right)^{2} \phi^{4}
\end{aligned}
$$

Then, while the unbounded from below potential remains approximately the same, the coefficients of the bounding two loop components are modified. However, the change in the two loop coefficients in the general model can be expected to be small because the weak and electromagnetic couplings are much smaller than the strong coupling at the chosen $\mu=11.63$ $\mathrm{GeV}$ scale. This property indicates that the form of the one and two loop terms of the simple model considered here should approximately resemble the result in the modified full SM in which the quadratic in the Higgs field terms is changed in sign respect to the one in the usual SM. Then, at this point it can be noticed that, since the one and two loop terms should be similar, the results for the Higgs mass in the model, which resulted smaller than the experimental Higgs mass, becomes a positive conclusion rather than a negative one. This comes from the fact that if the evaluation of the mass contributions would result to be larger than the Higgs mass, the added mass squared term $\frac{m^{2}}{2} \phi^{2}$ in the modified SM will become negative in order for the theory to reproduce the observed Higgs mass. Since the addition of a positive mass squared term is a natural choice for the starting Lagrangian, the model allows to conclude that the consideration of the "second minimum" to generate the spontaneous symmetry breaking in the modified SM to be considered, opens the possibility of fixing the observed value of the Higgs mass. In addition, since only one minimum can be expected to appear, the proposed model have the chance of eliminating the presence of instability in the SM.

Let us finally comment on the renormalization point chosen. In order to fix the observed top mass, we selected the scale at constant coupling of the value $\mu=11.63 \mathrm{GeV}$. Then, it should be checked that after implementing the renormalization group invariance, the running values of the parameters evaluated in the more standard renormalization point at $\mu=90 \mathrm{GeV}$, can reproduce the measured mass and coupling parameters at this point. But, such a possibility seems to be allowed by the freedom in the parameters in the modified SM proposed. In this sense, we note that we have chosen to fix the observed Top quark mass value at the relatively small scale $\mu=11.63 \mathrm{GeV}$. This is simply an option that we employed, however, we could also had considered that the Top quark mass could be increased in reducing the scale $\mu$ and use the renormalization group evolution to reproduce the observed parameters at $\mu=90 \mathrm{GeV}$. These possibilities are expected to be examined in the planned extension of the work. 


\section{SUMMARY}

We have explored the possibility that the spontaneous symmetry breaking effect in the SM could be implemented thanks to the Yukawa interaction of the Top quark with the Higgs field. For this purpose a formerly proposed simple model was reconsidered. The previous work although indicating in some sense the possibility investigated, was inconclusive due to the arising in it of a small value of the renormalization scale (smaller than 1 $\mathrm{GeV}$ ) in order to allow the Top quark to get the observed value of its mass.

In the present work, we evaluated again the effective potential created by the system for the scalar field, and found calculation errors. Their correction then, led to a picture in which it is also possible to fix the Top quark mass value, but at an intermediate value of the scale $\mu=11.63 \mathrm{GeV}$. The value of the scalar particle mass now emerging was of nearly $45 \mathrm{GV}$ which is close in order but smaller than the Higgs mass. However, being an amount smaller still allows for the possibility that in a modified SM considering a positive mass squared term for the Higgs field the observed Higgs mass of $126 \mathrm{GeV}$ can be fixed.

The work also investigates the stability of the result for the spontaneously symmetry pattern by considering the effect of employing the running with momentum coupling in the calculation. For this purpose, the finite expression in dimensional regularization of the quark-gluon contribution two loop effective potential for the Higgs fields was expressed as a momentum integral through a specially designed substraction procedure. The difficulty in attaining this formula, was produced by the use of dimensional regularization. In this scheme, the divergences are normally substracted after integrating over the momenta. However, we required to conserve the momentum integral in order to allow the substitution of the constant coupling by the running one. Then a momentum integral was retained by first substracting to the momentum integrand a relatively simple expression, which makes the momentum integral finite. Afterwards, the integral that was substracted, was exactly evaluated in dimensional regularization. This allowed to determine the divergent pole part and also the finite part which is also dependent of a parameter just introduced for eliminating a remaining zero momentum divergence. The divergent part just reproduced the minimal substraction counterterm of the quarkgluon contribution to the two loop effective action. Finally, the mentioned additional parameter was fixed by imposing that the finite part of the substracted integral vanishes for all values of the scalar field and scale parameter.

Further, the obtained formula for the potential (influenced by substituting the running coupling) was calculated for a close value of the scale parameter for which the potential was earlier evaluated at a constant coupling. The formula for the running coupling employed was a fit to the available data for the measured couplings. The values of the coupling at small momenta were assumed to be constant when the momentum value is smaller than the one associated to the maximal value of the measured coupling. The results for the potential became modified with respect to the ones evaluated for constant couplings at the same scale. However, after step by step reduction of the scale parameter $\mu$, the minimum of the potential was again fixed at the value defining the top quark mass for the fermion of the model. The resulting value of the scale was $4.95 \mathrm{GeV}$ which determines coupling of $\alpha=\frac{g^{2}}{4 \pi}=0.216$, which is still relatively small and being included in the region of measured interaction constants. This outcome allows to conclude that the reduction of the coupling with momenta still suggests the possibility for the occurrence of the examined spontaneous symmetry breaking pattern.

In a future extension of the work, we plan to start from a Lagrangian being practically equivalent to the SM's one, in which all the Higgs field terms associated to the usual scalar doublet will be present, but in which only the negative mass squared term creating the Mexican Hat potential will not be considered. The idea will be to attempt to use the various parameters in this slightly modified SM model, for implementing a symmetry breaking patterns being similar to the one discussed here.

\section{ACKNOWLEDGEMENTS}

The funding support of the Office of External Activities (OEA) of the International Centre for Theoretical Physics (ICTP), under its Network-35 is greatly acknowledged. Also, A.C. would like to acknowledge a helpful discussion with Prof. Masud Chaichian in which he underlined the motivating possibility of checking whether the use of the running coupling constant in place of a constant coupling would support the spontaneous symmetry breaking pattern.

\section{References}

[1] M. Gonderinger, Y. Li, H. Patel and M. Ramsey-Musolf, J. High Energy Phys. 1001, 053 (2010)

[2] J.A. Casas, J.R. Espinosa and M. Quiros, Phys. Lett. B 382, 374 (1996)

[3] C.D. Froggatt, H.B. Nielsen, Phys. Lett. B 368, 96 (1996)

[4] A. Cabo, Eur. Phys. J. C 71, 1620 (2011)

[5] T. Muta, Foundations of Quantum Chromodynamics, World Scientific Lectures Notes in Physics, vol. 5 (1987)

[6] H. Arason, D.J. Castaño, B. Kesthelyi, S. Mikaelian, E.J Piard, P. Ramond and B.D. Wright, Phys. Rev. 46, 3945 (1992)

[7] J. Fleischer and O.V. Tarasov, Z. Phys. C 64, 413 (1994)

[8] H. Bateman and A. Erdelyi, Higher Trascendental Functions, vol. 1, McGraw-Hill, New York (1953)

[9] M.J. Schlosser, Multiple Hypergeometric Series-Appell Series and beyond, arXiv:1305.1966v1 (2013)

[10] Review of Particle Physics, Journal of Physics G: Nuclear and Particle Physics, Vol. 33 Pp. 1-1232 (2006) 